\title{
Constructing a database of computational methods for environmental acoustics
}

\author{
Toru Otsuru ${ }^{1, *}$, Tetsuya Sakuma ${ }^{2, \dagger}$ and Shinichi Sakamoto ${ }^{3, \ddagger}$ \\ ${ }^{1}$ Faculty of Engineering, Oita University, \\ 700 Dannoharu, Oita, 870-1192 Japan \\ ${ }^{2}$ Institute of Environmental Studies, University of Tokyo, \\ 7-3-1 Hongo, Bunkyo-ku, Tokyo, 113-0033 Japan \\ ${ }^{3}$ Institute of Industrial Science, University of Tokyo, \\ 4-6-1 Komaba, Meguro-ku, Tokyo, 153-8505 Japan
}

(Received 6 September 2004, Accepted for publication 23 October 2004 )

Keywords: Computational room acoustics, Wave based method, World wide web database

PACS number: 43.55.Ka [DOI: $10.1250 /$ ast.26.221]

\section{Introduction}

Recent years have witnessed the widespread application of numerical methods such as FEM, BEM, and FDM to acoustical problems. Furthermore, various new related techniques are also being developed. Nevertheless, software users are sometimes confused when choosing an appropriate method among them because clear indications are lacking in regard to choice. For example, one researcher may implement a new method, but cannot sufficiently compare the numerical result with those obtained by other methods. One reason for this situation is that there have been few collaborative opportunities that allow mutual comparison of their results.

A suite of benchmark problems is arranged on the world wide web site based on a study by the authors [1] to allow the mutual comparison of computational accuracy and efficiency of various methods. A specific form for contribution of numerical results is also provided below to clarify the comparison. The web site is constructed and run by the working group on "Computational Methods for Environmental Acoustics, Architectural Institute of Japan." This paper is a revised article based on the authors' previous reports $([2,3])$.

\section{Platform and benchmark problems}

The database on the web site consists of five parts: "Top," "Introduction," "Directions," "Benchmark Problems" and "Papers," to provide a useful benchmarking platform. Its main language is English; the Japanese pages are provided as a supplement.

Table 1 shows the latest version of benchmark problem classifications; the problems share the main part of the platform. The table is provided on the "Introduction" page of the web site.

\subsection{Basic regulations}

Basic regulations for the calculation of each benchmark problem are given on the "Directions" page; the air condition, time factor, and units are determined as general regulations (see Table 2). Other regulations are assigned individually for respective problems in the frequency domain and time

\footnotetext{
*e-mail: otsuru@cc.oita-u.ac.jp

†e-mail: sakuma@k.u-tokyo.ac.jp

†e-mail: sakamo@iis.u-tokyo.ac.jp
}

domain.

In frequency domain problems, a stationary state with a single frequency is considered where octave-band center frequencies are assigned ranging from $31.5 \mathrm{~Hz}$ to $4 \mathrm{kHz}$. In time domain problems, the sampling frequency is fixed at $8 \mathrm{kHz}$. If a filtered pulse is used as the source, it must be mentioned in the data file described below. If supplementary data are necessary, they must also be noted.

\subsection{Categories and types}

The benchmark problems on the platform are classified as either: A - exterior; B - interior; or C - structuralacoustic. Each category has two types of problems: $0-$ basic and 1 - practical.

To construct the basic problems, simple objects are arranged individually: a cube, a sphere, and a flat panel. They are designated with the index number " 0 ," or as $\mathrm{A} 0$, B0, or C0. Similarly, regarding practical problems and realistic objects, an irregularly shaped reverberation room contains a loud speaker, a panel diffuser, a noise barrier, and an auditorium, which are designated with index numbers: "1," or $\mathrm{A} 1, \mathrm{~B} 1$ and $\mathrm{C} 1$.

To identify the problem properties clearly, numbers and symbols are added to the index with hyphenation, or A0-1F. Typical examples of the two types, basic and practical, are illustrated in Figs. 1 and 2.

\subsection{Tasks}

If necessary, several "Tasks" can be attributed to each problem to clarify details of the problem's computing conditions or settings. For example, B0-1F has two tasks: A and B. The former is to compute "Spatial distribution of sound pressure amplitude [Pa] at 101 points (R1), on the line $x=z=0.5$, which is located at $0.01 \mathrm{~m}$ intervals $(y=$ $-0.5,-0.49,-0.48, \ldots, 0.5)$ at the octave-band center frequencies $(f=31.5 \mathrm{~Hz}, 63 \mathrm{~Hz}, 125 \mathrm{~Hz}, 250 \mathrm{~Hz}, 500 \mathrm{~Hz}, 1 \mathrm{kHz}$, $2 \mathrm{kHz}$, and $4 \mathrm{kHz}$ ), while the latter computes "Frequency response of sound pressure amplitude $[\mathrm{Pa}]$ at three points $(0.5$, $0.5,-0.5)(\mathrm{R} 2),(0,0,0.5)(\mathrm{R} 3)$ and $(0.4,0.3,0.2)(\mathrm{R} 4)$; at frequencies with $1 \mathrm{~Hz}$ intervals from $20 \mathrm{~Hz}$ to $4 \mathrm{kHz}$, or with $10 \mathrm{~Hz}$ intervals from $20 \mathrm{~Hz}$ to $4 \mathrm{kHz}$."

As subsidiaries for the preprocessing of a computation, datasets of geometrical frames and meshes are arranged for problems with complicated shapes. 
Table 1 Classification of benchmark problems.

\begin{tabular}{|c|c|c|c|c|c|}
\hline Category & Type & Index & Domain & Object & Note \\
\hline \multirow[t]{18}{*}{ A. exterior } & \multirow[t]{9}{*}{ 0. basic } & $\mathrm{A} 0-1 \mathrm{~F}$ & frequency & vibrating cube & radiation \\
\hline & & $\mathrm{A} 0-2 \mathrm{~F}$ & frequency & cube & radiation \\
\hline & & $\mathrm{A} 0-2 \mathrm{~T}$ & time & cube & radiation \\
\hline & & $\mathrm{A} 0-3 \mathrm{~F}$ & frequency & sphere with a point source & scattering \\
\hline & & A0-3T & time & sphere with a point source & scattering \\
\hline & & $\mathrm{A} 0-4 \mathrm{~F}$ & frequency & sphere & scattering \\
\hline & & A0-4T & time & sphere & scattering \\
\hline & & $\mathrm{A} 0-5 \mathrm{~F}$ & frequency & flat panel & diffraction \\
\hline & & $\mathrm{A} 0-5 \mathrm{~T}$ & time & flat panel & diffraction \\
\hline & \multirow[t]{9}{*}{ 1. practical } & $\mathrm{A} 1-1 \mathrm{~F}$ & frequency & loudspeaker & radiation \\
\hline & & A1-1T & time & loudspeaker & radiation \\
\hline & & A1-2F & frequency & diffuser & scattering \\
\hline & & A1-2T & time & diffuser & scattering \\
\hline & & A1-3F & frequency & barrier on the ground & diffraction \\
\hline & & A1-4F & frequency & rigid barrier with cylindrical edge & diffraction \\
\hline & & A1-4T & time & rigid barrier with cylindrical edge & diffraction \\
\hline & & A $1-5 \mathrm{~F}$ & frequency & absorptive barrier with cylindrical edge & diffraction \\
\hline & & A1-5T & time & absorptive barrier with cylindrical edge & diffraction \\
\hline \multirow[t]{8}{*}{ B. interior } & \multirow[t]{2}{*}{ 0. basic } & B0-1F & frequency & cubic cavity & closed cavity \\
\hline & & B0-1T & time & cubic cavity & closed cavity \\
\hline & \multirow[t]{6}{*}{ 1. practical } & $\mathrm{B} 1-1 \mathrm{~F}$ & frequency & auditorium & closed cavity \\
\hline & & B1-1T & time & auditorium & closed cavity \\
\hline & & B1-2F & frequency & auditorium with seats & closed cavity \\
\hline & & $\mathrm{B} 1-2 \mathrm{~T}$ & time & auditorium with seats & closed cavity \\
\hline & & B1-3F & frequency & reverberation room & closed cavity \\
\hline & & B1-3T & time & reverberation room & closed cavity \\
\hline \multirow{3}{*}{$\begin{array}{l}\text { C. structural- } \\
\text { acoustic }\end{array}$} & 0 . basic & $\mathrm{C} 0-1 \mathrm{~F}$ & frequency & plate in baffle & insulation \\
\hline & 1. practical & $\mathrm{C} 1-1 \mathrm{~F}$ & frequency & plate in a room & radiation \\
\hline & & $\mathrm{C} 1-2 \mathrm{~F}$ & frequency & plate between rooms & insulation \\
\hline
\end{tabular}

Table 2 Basic regulations for calculation.

\begin{tabular}{ll}
\hline Time factor & $\exp (+i \omega t)$ \\
\hline Air condition & \\
& $c=343.7 \mathrm{~m} / \mathrm{s}$ \\
& $\rho=1.205 \mathrm{~kg} / \mathrm{m}^{3}$ \\
& $T=20^{\circ} \mathrm{C}$ \\
\hline
\end{tabular}

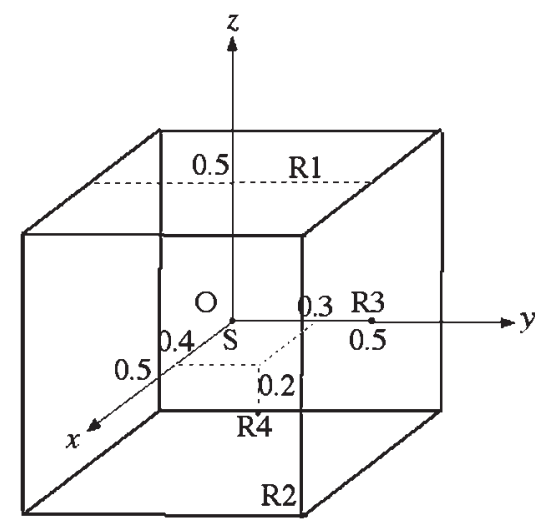

Fig. 1 Geometry of problem $(\mathrm{B} 0-1 \mathrm{~F} / \mathrm{T})$ with a point source (S) and receiving points (R1-R4). Unit [m].

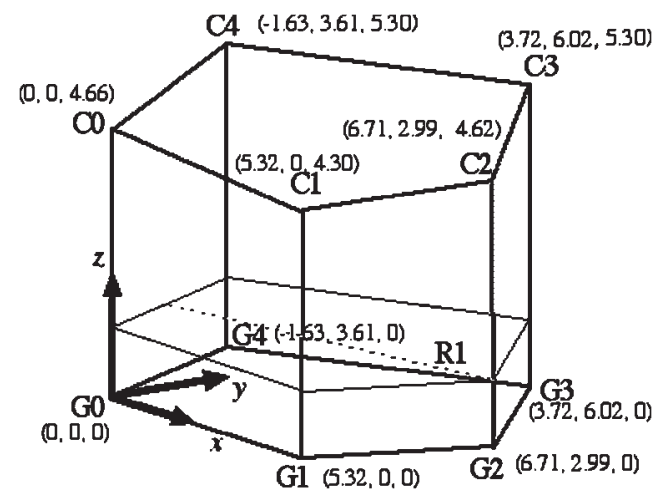

Fig. 2 Geometry of problem (B1-3F/T). A point source (S) and receiving points (R1). R1 comprises 53 points on a line. Unit $[\mathrm{m}]$.

The CPU-time and used memory are measured and specified to evaluate computational efficiency. Note that the CPU-time does not include the time for mesh generation. Regarding additional information of a computation, such as applied mesh division, element type(s) and size(s), the number of nodes, degrees of freedom, and so on should be specified in the data file, as shown below. 


\subsection{Data files for contribution}

A downloadable MS-Excel formatted file is provided for contributors to list their computed results. The file comprises three sheets: face items, numerical output, and computing performance. All supplementary information about a contributor's computation can be filled into the Excel-cell that is prearranged as that for "Comments" on each sheet. The data file, which is submitted to the web-administrator, is to be included in the "Contributions" section, where it is represented by the method name and contributor.

\section{Example applications}

Using the database, one can compare results with those obtained by other contributors listed in the data files. Figure 3 shows an example of a comparison of results of $\mathrm{B} 0-1 \mathrm{~F}$ as computed by modal summation, FEM, BEM and FMBEM. In the data files, explanations of each computational environment are given in the face items. Table 3 shows several

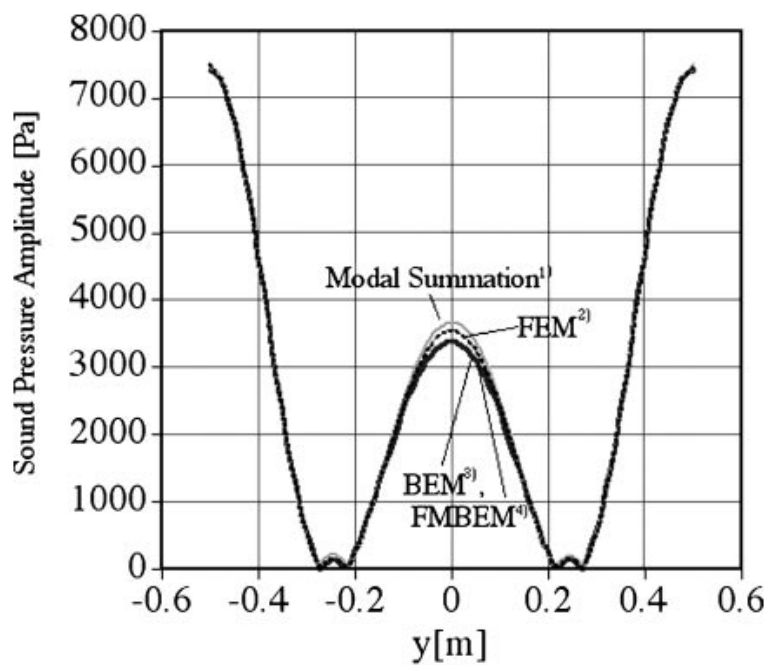

Fig. 3 Example application of the database. Results are compared for B0-1F computed by Modal-Summation, FEM, BEM, and FMBEM. Symbol superscripts correspond to the numbers in Table 3.
Table 3 Summary of face items submitted to B0-1F.

\begin{tabular}{ccc}
\hline No. & Method & Machine \\
\hline 1 & Modal Summation & iMac PowerPC G3 \\
2 & FEM & SunBLADE 2 x UltraSPARCIIICu \\
3 & BEM & Dell Precision WorkStation 610 \\
4 & FMBEM & ditto \\
\hline
\end{tabular}

exemplary items that are selected from downloaded sheets.

Regarding practical problems, an analytical solution is not obtained easily. In such cases, measured data are valuable as a reference. In this respect, some contributors have submitted their computational results with measured data. An example of one case is given in Fig. 4. The figure shows a comparison between sound pressure levels as computed by FEM and measured data on B3-1F at $1 / 3$ octave-band centered on $250 \mathrm{~Hz}$. Pearson's correlation coefficient, $\mathrm{R}$, is equal to 0.89 in this case. Since, in general, a measurement at a single frequency is not easy to give meaningful sound pressure value, octave or 1/3 octave-band measurements are usually conducted. In such cases, computed values at the frequencies within the band width must be integrated to give a single number.

\section{Concluding remarks}

This paper introduced a world wide web database of computational methods for environmental acoustics along with several example applications. The project has just begun; contributions from various aspects of numerical methods including conventional techniques are welcomed. The progress of computing power is sufficiently rapid that extension of current computational methods is also expected. The web site address is: http://gacoust.hwe.oita-u.ac.jp/AIJ-BPCA/.

\section{Acknowledgments}

The authors would like to acknowledge the continuous cooperation of both WG member and nonmember contributors. This work was supported by JSPS, a Grant-in Aid for Scientific Research (A) 15206064, 2003-4.

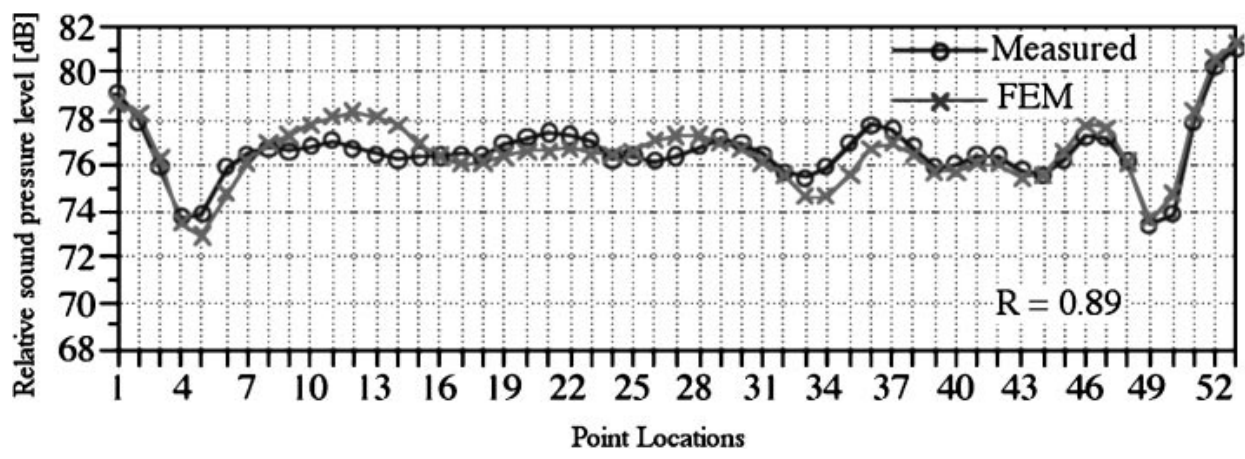

Fig. 4 Comparison of sound pressure level distribution in a reverberation room (B3-1F) between FEM and measurement at $1 / 3$ octave-band centered on $250 \mathrm{~Hz}$. 


\section{References}

[1] T. Sakuma, P. Svensson, A. Franck and S. Sakamoto, "A roundrobin test on wave-based computational methods for roomacoustic analysis," Proc. Forum Acusticum Sevilia 2002, NUM-02-001-IP (2002).
[2] T. Sakuma, S. Sakamoto and T. Otsuru, "A benchmarking framework for wave-based computational methods in architectural acoustics," Proc. ICA2004, Vol. I, 281-284 (2004).

[3] T. Otsuru, T. Sakuma and S. Sakamoto, "Activities for constructing database on computational methods for environmental acoustics," Proc. RADS2004, CD-ROM (2004). 